\title{
Estudio sobre la implementación de un sistema de información clínico en Honduras (SICHO)
}

Elder David Godoy García ${ }^{1}$

Yeny Carías ${ }^{2}$

\section{RESUMEN}

En esta investigación se propone un sistema de información clínico en Honduras (SICHO), el cual administrará las citas médicas, el historial clínico a nivel nacional y control de medicamentos en los centros médicos públicos.

Dicho sistema brindará información del paciente: historial de alergias, visitas médicas recetas anteriores, tratamientos; además, brindará información estadística exacta de posibles epidemias. También se estudiará la viabilidad económica del sistema sanitario del país, para la aplicación de dicho sistema a nivel nacional.

La toma de datos de los posibles beneficiados (pacientes de un centro médico), ha dado como resultado que están de acuerdo en que traería grandes beneficios si se realiza este sistema. Los médicos entrevistados están a favor, ya que les facilitaría el estudio de los casos y control en sus clínicas de datos de sus pacientes; así, a pesar de la renuencia al cambio en los médicos, la mayoría está de acuerdo en que les ayudaría a sacar provecho de toda la información previamente capturada por sus colegas.

Palabras clave: sistema, medicina, Honduras, clínica, control, estudio.

${ }^{1}$ Estudiante de la Carrera de Ingeniería en Sistemas, Facultad de Ingeniería, UNAH: eldergodoy54@gmail.com

${ }^{2}$ Asesora, Carrera de Ingeniería en Sistemas, Facultad de Ingeniería, UNAH: yenycarias@unah.edu.hn 


\section{ABSTRACT}

In this research, a clinical information system is proposed in Honduras (Sicho), created for the administration of medical appointments, medical history and national drug control in public medical centers.

This system will provide patient information: a history of allergies, past medical visit prescriptions, treatments; thus providing accurate statistical information of possible epidemics. It will also study the economic viability of the health system in the country, in order to implement such a nationwide system.

The data collection of potential beneficiaries (patients from one medical center), has resulted in the agreement of those who have benefited from the system. Interviewed doctors are in favor, because the system facilitates the study of cases and the patient control clinical data; so, despite the reluctance to change on behalf of the doctors, the majority agree that it would help them take advantage of all previous data recorded by their colleagues.

Keywords: system, medicine, Honduras, clinical control study. 


\section{INTRODUCCIÓN}

La falta de control de los historiales clínicos en el país ha causado muchas veces pérdidas económicas y sobretodo humanas, muchas veces en las emergencias se pierden vidas humanas por desconocimiento de las alergias o tratamientos que llevaba el paciente.

El sistema representa una ayuda para los médicos, que les permitiría evitar este tipo de tragedias y llevar control de los medicamentos, vacunas y tratamientos que le han dado al paciente a lo largo de su vida; en caso de que sea otro médico, este podrá contactarse con el médico que le recetó los medicamentos para poder llevar un estricto orden de todos los tratamientos.

También traería beneficios a los investigadores y estadistas del país, ya que tendrán al alcance toda la información necesaria para prevenir epidemias a nivel nacional y preparar las farmacias públicas con los medicamentos que necesitarán para enfrentar dichas enfermedades.

\section{MARCO TEÓRICO}

\section{Contexto}

Honduras tiene aproximadamente una población de 8 millones de personas, de las cuales el $54 \%$ vive en zonas urbanas; un $46 \%$ en zonas rurales, un $51 \%$ son mujeres y un $49 \%$ son hombres, según el XVII censo de población y VI de vivienda (2015).

Honduras cuenta con 28 hospitales públicos, en diferentes zonas del país, siendo el más antiguo el Hospital General San Felipe y el más grande el Hospital Escuela Universitario, todos administrados por la Secretaría de Salud.

Solo en Tegucigalpa existen 5 hospitales privados, 2 en San Pedro Sula, y en La Ceiba y Roatán 1; estos hospitales son reconocidos y recomendados por la Embajada de Estados Unidos de América. Por lo tanto, al existir aproximadamente 35 hospitales a nivel nacional, muchas veces con datos de los mismos pacientes en diferentes centros medicos, surge el problema de la duplicación y pérdida de información. 
Un sistema nacional de control de historiales clínicos conllevaría al ahorro de tiempo en los tratamientos de los pacientes en los diferentes hospitales, ya que este llevaría desde los datos más básicos como vacunas, tratamientos y alergias, hasta los más complejos qué han tratado al paciente, avisos de citas mediante correo electrónico (si cuenta con uno), o bien, mediante un mensaje texto a su número celular, datos de familiares para control de enfermedades hereditarias y control de farmacias en hospitales públicos (ubicados en un solo lugar).

Este sistema sería accesible para cualquier médico colegiado a nivel nacional y tendrá datos de los médicos que han tratado al paciente para mejorar la comunicación entre médicos y asegurar un mejor tratamiento al paciente, el cual muchas veces visita diferentes médicos y recibe diversos tratamientos; se incluirían hospitales privados para tener control de los pacientes por epidemias o emergencias que puede tener el mismo, dado que muchas veces al primer centro médico que llevan al paciente en una emergencia es el Hospital Escuela Universitario (ver figura 1).

\section{Figura 1. Hospital Escuela Universitario}

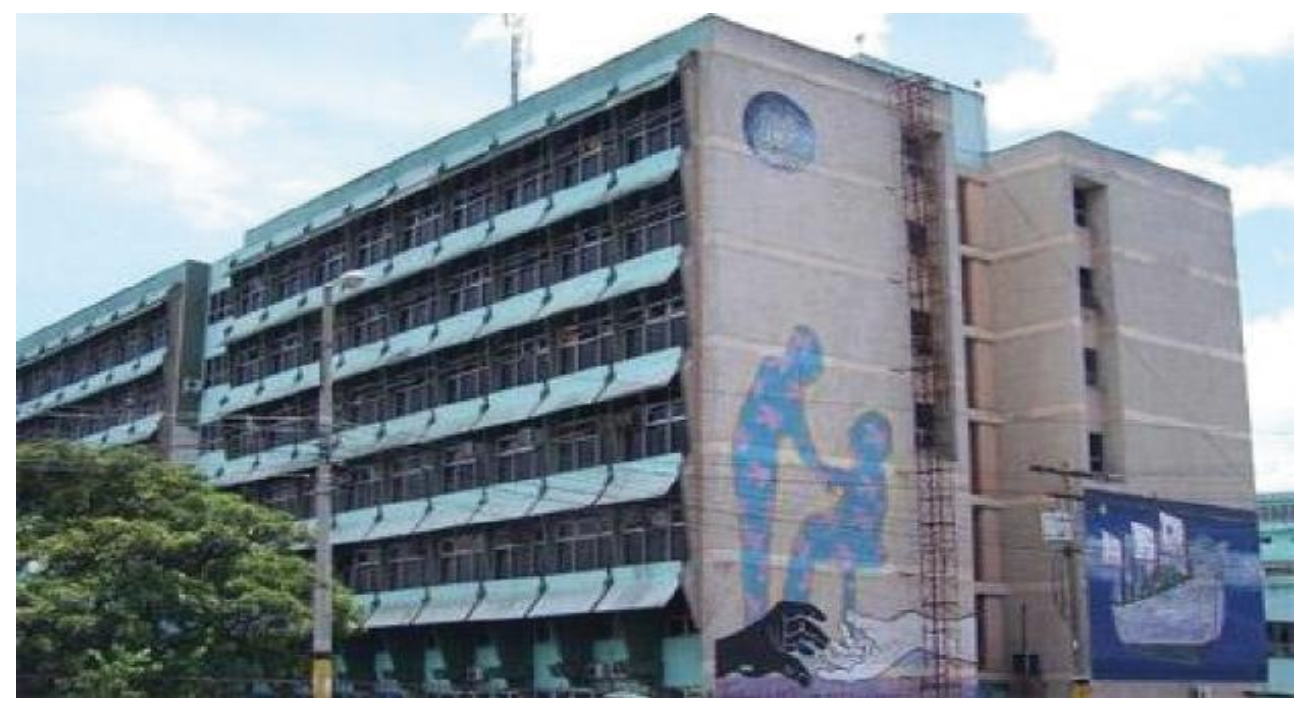

Fuente: Diario La Tribuna

También abarcaría a las farmacias privadas para llevar un control de los medicamentos que toma el paciente y verificar contra las indicaciones médicas en los diferentes medicamentos que las farmacias tienen. Se tiene planificado también que el paciente tenga acceso a sus datos, tales como citas médicas, información de medicamentos y tratamientos, para que tenga conocimiento y tome conciencia de su salud. 
Para identificar al paciente se usaría el número de identidad dado, pues este número es único en todo el país; o bien, la huella digital para cuando se realicen campañas de vacunación en las zonas rurales del país, en donde se dificulta la movilización de un computador, pero sí es posible hacerlo con un lector de huella digital.

En caso que el paciente sea extranjero, su identificador sería su pasaporte y se le pedirían datos claves a su país de origen para controlar posibles padecimientos crónicos o enfermedades infecciosas que tenga; de esta manera se puede estar preparado para patógenos no comunes en el país.

\section{Cuadro 1. Indicadores básicos, Honduras, 2009}

\begin{tabular}{lc|}
\hline Población total & $8,045,990$ \\
\hline Esperanza de vida & 72.1 \\
\hline Esperanza de vida a los 60 años (2008) & 13.7 \\
\hline Tasa de mortalidad general & 4.9 \\
\hline Tasa de mortalidad infantil & 27.8 \\
\hline Razón de mortalidad materna (2005) & 280 \\
\hline Prevalencia de diabetes (\%) (>19 años en zonas urbanas) & 6.2 \\
\hline Prevalencia de hipertensión arterial (\%) (>19 años en zonas & 22.6 \\
\hline urbanas) & \\
\hline Proporción de partos atendidos por personal calificado & 69 \\
\hline Cobertura de vacunación (\%) en niños de 12 a 23 meses & \\
\hline Tercera dosis DPT & 99 \\
\hline BCG niños de 1 año & 99 \\
\hline Sarampión niños de 1 año & 95 \\
Población cubierta por la seguridad social (\%) & 18 \\
\hline Población no asegurada (\%) & 79 \\
\hline Población con seguro privado (\%) & 2.9 \\
\hline Gasto total en salud como porcentaje del PIB (2008) & 5.7 \\
\hline Gasto en salud per cápita (2008) * & 227 \\
\hline Gasto público en salud como porcentaje del gasto total en & 61.4 \\
\hline salud (2008) & 8.7 \\
\hline Porcentaje de hogares con gastos catastróficos pormotivos \\
de salud (2004)
\end{tabular}

Fuente: Instituto Nacional de Estadísticas (2009), Trigésima Octava Encuesta de Hogares

* Dólares PPA (paridad de poder adquisitivo) 


\section{Cuadro 2. Diez principales causas de muerte, Honduras, 2004}

$\%$ muertes totales

1. Condiciones originadas en el periodo perinatal

2. Malformaciones congénitas y anormalidades cromosómicas

3. Diabetes mellitus

4. Enfermedad cerebrovascular

5. Eventos de origen no determinado

6. $\mathrm{VIH} / \mathrm{sida}$

7. Influenza y neumonía

8. Enfermedades pulmonares crónicas

9. Cirrosis y otras enfermedades del hígado

10. Enfermedades isquémicas del corazón

Fuente: Organización Panamericana de la Salud. Salud en las Américas.

Honduras. Washington, DC: OPS, 2007.

En el cuadro 1 se denota que la población no asegurada es mucho mayor a la asegurada, aunque muchas veces visitan diferentes centros de salud a lo largo del país, teniendo como problema la comunicación entre médicos.

En el cuadro 2 se pueden ver las principales causas de muerte en el país; así, en las enfermedades está la diabetes, que es una enfermedad hereditaria que puede ser controlada si el médico cuenta con la información de que en la familia del paciente hay casos de diabetes. Entre otras causas existen los eventos de origen no determinado, los cuales, con un buen control, a los investigadores de enfermedades les puede ayudar a mejorar sus estudios.

\section{MÉTODO}

Para la elección del método para el estudio se capturaron datos mediante una encuesta que permite el desarrollo de la investigación. La encuesta es cerrada, ya que la mayoría de las preguntas tienen respuestas determinadas, pero existe una pregunta que es semiabierta, esta ayuda a entender al paciente y sus experiencias en los diferentes centros médicos del país. Para determinar la muestra se toma en cuenta lo siguiente: variabilidad de personas que visitan los centros médicos a diario, 
nivel académico de las personas encuestadas y edad de las personas encuestadas.

Para determinar la muestra, se estableció un porcentaje de seguridad del $80 \%$ y un error precisión del $10 \%$, con una población de 8 millones de personas, lo que nos da una muestra aproximada de 41 personas a encuestar, tomadas al azar en diferentes centros médicos de Tegucigalpa, se concluyen los siguientes datos:

Donde $n$ es el valor de la muestra

$$
\begin{gathered}
Z=80 \% \text { con un } 1.8 \text { nivel de seguridad } \\
E=E l \text { margen de error de un } 10 \% \\
\text { P y } Q=0.50
\end{gathered}
$$

Por lo que:

$$
\begin{gathered}
\mathrm{n}=\left((1.28){ }^{\wedge} 2\right)^{*}(0.5) *(0.5) * 8,000,000 /\left(8,000,000 *((0.10) \wedge 2)+\left((1.28){ }^{\wedge} 2\right) *(0.5) *(0.5)\right) \\
\mathrm{N}=40.95 \approx 41 \text { personas de muestra }
\end{gathered}
$$

\section{ANÁLISIS DE DATOS}

\section{Edad:}

$\begin{array}{ll}15-24 & 27.1 \% \\ 25-34 & 60.4 \% \\ 35-44 & 6.3 \% \\ 45-54 & 2.1 \% \\ 55-64 & 4.2 \%\end{array}$

2.Sexo:

Masculino $77.1 \%$

Femenino $27.9 \%$ 
3.Zona donde vive:

Urbana $89.6 \%$

Rural 10.4

4. Visita un centro médico con regularidad:

Si $25 \%$

No $75 \%$

5. Tipo de médico que visita:

$\begin{array}{ll}\text { Público } & 35.4 \% \\ \text { Privado } & 64.6 \%\end{array}$

6. Si es público:

$\begin{array}{ll}\text { Hospital } & 43.8 \% \\ \text { Centro de salud } & 43.8 \% \\ \text { Clíper } & 12.5 \%\end{array}$

7. Si es privado es:

$\begin{array}{ll}\text { Hospital } & 10.4 \% \\ \text { Clínica } & 79.2 \% \\ \text { Particular } & 10.45 \%\end{array}$

8. ¿Tiene varios historiales clínicos en los diferentes centros médicos que ha visitdo?

Sí $\quad 58.3 \%$

No $\quad 41.7 \%$

9. ¿Le han perdido su historial clínico alguna vez?

Sí $\quad 27.1 \%$

No $\quad 72.9 \%$ 
10. ¿Alguna vez le han aplicado medicamento al cual usted es alérgico?

Sí $\quad 14.6 \%$

No $\quad 85.4 \%$

11. ¿Hay enfermedades hereditarias en su familia?

Sí $\quad 70.8 \%$

No $\quad 29.2 \%$

12.¿Le importaría que su historial clínico estuviera al alcance de cualquier médico colegiado?

Sí $\quad 47.9 \%$

No $\quad 52.1 \%$

13.¿Le importaría que sus datos clínicos sean usados para futuros estudios?

Sí $\quad 41.7 \%$

No $\quad 58.3 \%$

14.¿Le gustaría poder acceder a su historial clínico desde una computadora o dispositivo con acceso a internet?

Sí $\quad 95.8 \%$

No $4.2 \%$

15.¿Dónde cree que hay más pérdida de tiempo en su centro médico?

$\begin{array}{ll}\text { Farmacia } & 27.1 \% \\ \text { Sala de espera } & 85.4 \% \\ \text { Toma de datos } & 45.8 \% \\ \text { Otro } & 4.2 \%\end{array}$


16.¿Cree que el médico debería tener conocimiento de los medicamentos disponibles en la farmacia del centro médico?

Sí $\quad 93.8 \%$

No $\quad 6.3 \%$

17. A su parecer, las citas en el hospital público tardan:

$\begin{array}{ll}\text { Poco } & 0.4 \% \\ \text { Mucho } & 64.6 \% \\ \text { Demasiado } & 35 \%\end{array}$

18. ¿Cree que la implementación de un sistema de información clínico de Honduras (SICHO) le traería beneficios?
Sí $\quad 97.9 \%$
No $\quad 2.1 \%$

19. ¿Qué beneficios cree que tendría, si su número de identidad fuera su número de historial clínico nacional?

$\begin{array}{ll}\text { Ninguno } & 2.1 \% \\ \text { Poco } & 10.4 \% \\ \text { Bastante } & 97.5 \%\end{array}$

\section{RESULTADOS}

En la pregunta 5 se puede observar que solo un $35 \%$ de la muestra visita centros públicos con regularidad, ya que desconfían del sistema de salud pública por diferentes motivos, por ejemplo, descontrol y mala organización dentro de ellos; de manera que con un sistema de control todo sería transparente y rápido.

En la pregunta 8 se deduce que los pacientes tienen historiales clínicos en diferentes centros médicos, ya sea privados o públicos, muchas veces con diferentes tratamientos para la misma enfermedad. 
En la pregunta 9 se valora que a muy pocas personas se le ha perdido su historial clínico, pero a estas personas que se les perdió les ha causado muchos problemas con los diferentes tratamientos.

En la pregunta 11 se ve que la mayoría de las personas que se encuestaron sufre de enfermedades hereditarias, pero muchas pueden ser prevenidas desde temprana edad si se tiene el conocimiento de ello, esto ayudaría a los expertos en estadísticas a llevar un mejor control de dichas enfermedades.

Se puede determinar que las citas en los hospitales públicos se tardan mucho (pregunta 17), esto mejoraría con un sistema de citas, el cual determinaría la gravedad de la enfermedad y le asignaría una cita lo más pronto posible de acuerdo a sus necesidades.

Aunque muchas personas desconozcan las nuevas tecnologías y los beneficios totales que puede conllevar, la mayoría (pregunta 18) cree que si se implementa un sistema de información clínico en el país, los beneficios serían muchos y mejorarían el sistema de salud del país.

\section{¿Quiénes son los beneficiarios?}

Pacientes de centros médicos, hospitales, clínicas privadas y públicas, médicos e investigadores de enfermedades y epidemias, la Secretaría de Salud, las ONG que ayudan y brindan campañas médicas en el país y muchas veces no saben si los medicamentos que proporcionan al paciente le puede causar una afección.

\section{CONCLUSIONES}

La implementación de este sistema de información mejoraría mucho la atención en todos los centros médicos del país, sobre todo incluyendo a los centros médicos privados, para así lograr una centralización de todos los datos clínicos de todo el país. Esto también traería beneficios de control de inventario, puesto que en los centros médicos públicos muchas veces no se maneja un inventario de todos los medicamentos o de los materiales que se utilizan en él y con dicho sistema el médico tendría conocimiento de todos los medicamentos disponibles en las farmacias. 


\section{AGRADECIMIENTOS}

A Yeny Carías, por sus consejos y tutorías; igualmente a Natalia Gallo, por la capacitación en CITAVI. Gracias, también, a las personas que ayudaron con la encuesta en los diferentes centros médicos del país.

\section{BIBLIOGRAFÍA}

Instituto Nacional de Estadísticas. (2009). Trigésima octava encuesta de hogares. Tegucigalpa.

Organización Panamericana de la Salud. (2007). Salud en las Américas. Washington D.C.

Instituto Nacional de Estadísticas (2010) Comunicado de proyecciones poblacionales. Recuperado de: http://www.ine-hn.org/

Instituto Nacional de Estadísticas (2009) Trigésima Octava Encuesta de Hogares. Tegucigalpa: Instituto Nacional de Estadísticas. 